\title{
Human-in-the-Loop Investigation of Interoperability Between Terminal Sequencing and Spacing, Automated Terminal Proximity Alert, and Wake-Separation Recategorization
}

\author{
Todd J. Callantine, ${ }^{*}$ Nancy Bienert, ${ }^{\dagger}$ AbhayBorade,${ }^{\dagger}$ Conrad Gabriel,${ }^{\dagger}$ \\ VimmyGujral, ${ }^{\dagger}$ Kim Jobe, ${ }^{\dagger}$ Lynne Martin, ${ }^{\S}$ Faisal Omar ${ }^{\star}$ \\ San Jose State University/NASA Ames Research Center, Moffett Field, CA, 94035 \\ Thomas Prevot ${ }^{*}$ and Joey Mercer ${ }^{* *}$ \\ NASA Ames Research Center, Moffett Field, CA, 94035
}

\begin{abstract}
A human-in-the-loop simulation study addressed terminal-area controller-workstation interface variations for interoperability between three new capabilities being introduced by the FAA. The capabilities are Terminal Sequencing and Spacing (TSAS), Automated Terminal Proximity Alert (ATPA), and wake-separation recategorization, or 'RECAT.' TSAS provides controllers with Controller-Managed Spacing (CMS) tools, including slot markers, speed advisories, and early/late indications, together with runway assignments and sequence numbers. ATPA provides automatic monitor, warning, and alert cones to inform controllers about spacing between aircraft on approach. ATPA cones are sized according to RECAT, an improved method of specifying wake-separation standards. The objective of the study was to identify potential issues and provide recommendations for integrating TSAS with ATPA and RECAT. Participants controlled arrival traffic under seven different display configurations, then tested an 'exploratory' configuration developed with participant input. All the display conditions were workable and acceptable, but controllers strongly preferred having the CMS tools available on Feeder positions, and both CMS tools and ATPA available on Final positions. Controllers found the integrated systems favorable and liked being able to tailor configurations to individual preferences.
\end{abstract}

\section{Introduction}

$\mathrm{R}$ EDUCING tactical radar vectoring and step-down descents while providing precise minimum safe spacing on approach are essential for improving the efficiency of terminal-area operations during periods of high traffic demand. NASA's Air Traffic Management Technology Demonstration \#1 (ATD-1) activity ${ }^{1}$ addressed the first enabler by developing and transferring Terminal Sequencing and Spacing (TSAS) technology to the FAA. TSAS extends the metering and sequencing capabilities of the FAA's Time-Based Flow Management (TBFM) system into the terminal area. With TSAS, controllers use tools called Controller-Managed Spacing (CMS) tools to merge and space aircraft according to a schedule using primarily speed adjustments, thereby limiting the use of vectors and altitude clearances that interrupt efficient Performance-Based Navigation (PBN) arrivals (Fig. 1). ${ }^{2}$ The CMS tools are integrated with the FAA's Standard Terminal Automation Replacement System (STARS) displays, and include slot markers, speed advisories, and early/late indications. TSAS also provides runway and sequence number indications in aircraft full data blocks (FDBs) on STARS displays.

To enable minimum safe approach spacing, the FAA is also introducing two other terminal-area improvements. First, Automated Terminal Proximity Alert (ATPA) is designed to increase controller awareness of aircraft spacing

\footnotetext{
*Research Engineer, Human Systems Integration Division, NASA Ames Mail Stop 262-4, AIAA Senior Member.

${ }^{\dagger}$ Research Associate, Human Systems Integration Division, NASA Ames Mail Stop 262-4.

*Research Engineer, Human Systems Integration Division, NASA Ames Mail Stop 262-4.

${ }^{\S}$ Research Pyschologist, Human Systems Integration Division, NASA Ames Mail Stop 262-4.

${ }^{* *}$ Research Psychologist, Human-Systems Integration Division, NASA Ames Mail Stop 262-4, AIAA Member.
} 


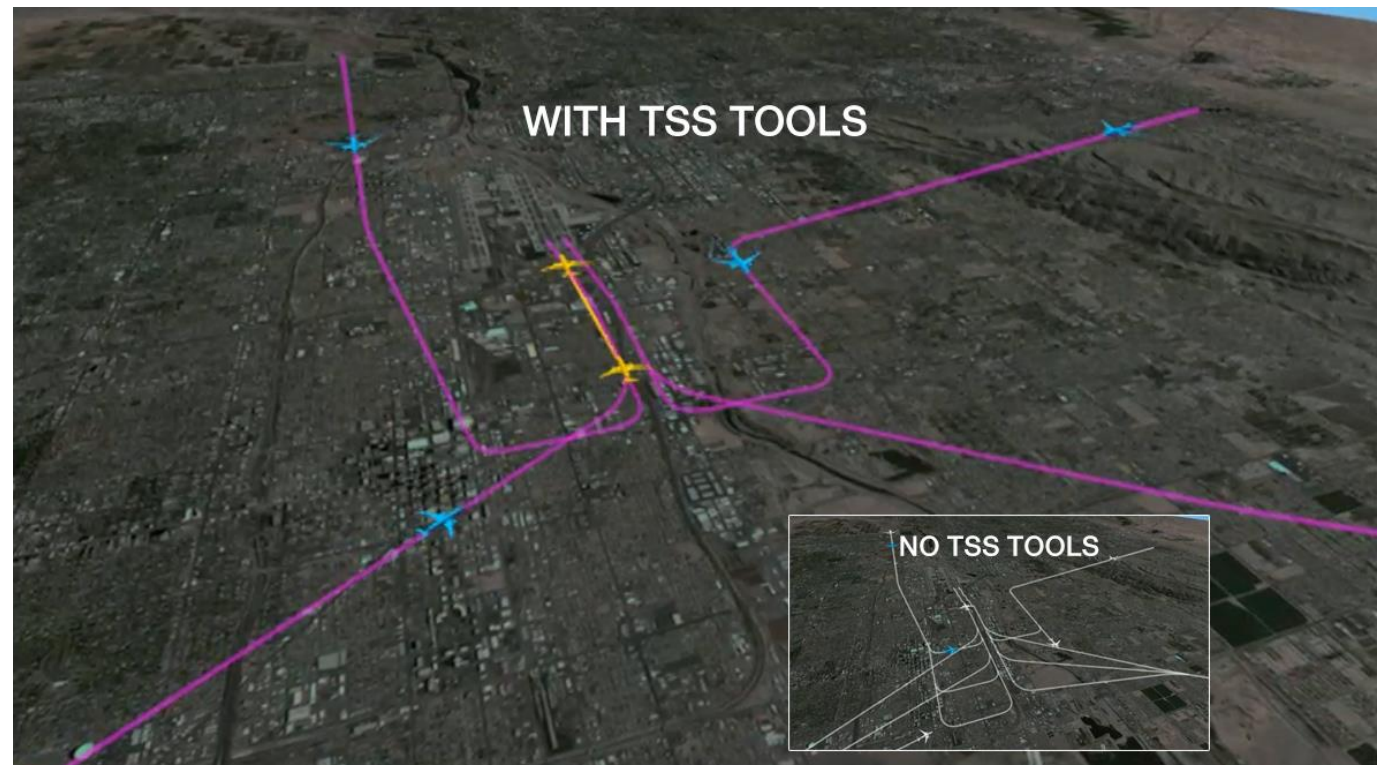

Figure 1. PBN route conformance with TSAS (formerly referred to as 'TSS' during ATD-1 development).

in a speed-control environment. ATPA extends existing Terminal Proximity Alert (TPA) functionality so that monitor, warning, and alert cones appear automatically for aircraft on approach when certain conditions are met. Second, wake-separation recategorization, or 'RECAT,' is a new method of specifying wake-separation standards based on aircraft wingspan, speed, and stability characteristics in addition to weight. RECAT increases the number of wake-spacing categories and allows tighter spacing between certain pairs of aircraft than current standards without compromising safety.

An ATD-1 human-in-the-loop simulation was conducted in the Airspace Operations Laboratory (AOL) at NASA Ames Research Center in June 2015 to investigate controller-interface variations and interoperability between TSAS, ATPA, and RECAT in support of the FAA/NASA Operational Integration Assessment (OIA) for the TSAS system prototype. The study sought to identify potential issues and provide integration recommendations. Two teams of terminal-area controllers worked simulated west-flow arrivals in the Phoenix TRACON (P50) in parallel. Traffic included a mix of jets and turboprops scheduled with TBFM using staggered dependent-runway and nonstaggered independent-runway configurations. Equipped aircraft were assigned to RNP approaches and aircraft could be scheduled as 'crossovers' to the parallel runway. During the first three days of the study, participants evaluated TSAS and the CMS tools, ATPA, and RECAT in seven different configurations; an additional exploratory condition created using participant input was tested on the final day of the study. Data collected via post-trial and end-of-study questionnaires, controller workload prompts, and debrief sessions, along with $\mathrm{A} / \mathrm{V}$ recordings and digital data, were used to assess controller usage and preferences. Integration recommendations were formulated and presented to the FAA based on the study results. Before describing the study and results, the following subsections provide additional details about the CMS tools, ATPA, and RECAT.

\section{A. Controller-Managed Spacing Tools}

The CMS tools provide terminal-area controllers with knowledge of the TSAS schedule and information helpful for controlling aircraft to meet the schedule. TSAS is designed for use in a TBFM arrival-metering environment in which center controllers have preconditioned arrivals, so that aircraft arrive in the terminal area close to onschedule. In the TRACON, circular targets on the STARS display called slot markers translate the schedule information into salient spatial cues. At any given time, the slot marker for a particular aircraft displays where the aircraft should be if it flies its assigned PBN arrival through the forecast wind field, meeting the speed and altitude profile for the published PBN procedure adapted in TBFM, and arrives at its Scheduled Time of Arrival (STA) at the merge point or runway threshold. ${ }^{3}$ Thus, a slot marker provides a continuous graphical depiction of an aircraft's progress relative to the TSAS terminal-area schedule.

Slot-marker diameter is specified in terms of time ( $15 \mathrm{~s}$ is a typical value), so that the slot markers decrease in diameter as their current speed decreases along the arrival profile. The slot marker speed is displayed next to the slot marker and the aircraft's current indicated airspeed is displayed next the the aircraft target symbol (Fig. 2). Dwelling 
on an aircraft's FDB highlights its slot marker. If CMS schedule timelines are also shown on the STARS display, dwelling on a FDB also highlights the corresponding callsign on the timeline; similarly, dwelling on a timeline callsign highlights the aircraft's FDB along with its slot marker.

The CMS tools also include FDB indications for the TSAS assigned runway and sequence number, as well as speed advisories or early/late indications. These values time-share in the third line of the FDB (Fig. 3). A speed advisory appears in the third line of an aircraft's FDB when its Estimated Time of Arrival (ETA) differs from its STA by more than an adapted value (e.g., $5 \mathrm{~s}$ ) and only if the predicted speed will correct the difference; otherwise, the early/late indicator is displayed (see Fig. 2). Speed advisories are suggested airspeeds intended only to provide guidance for controllers about speed clearances for adjusting aircraft toward their STAs; the TSAS functionality integrated into TBFM predicts that flying the advised speed until rejoining the nominal $\mathrm{PBN}$ speed profile will place the aircraft on schedule. In addition, for equipped aircraft assigned to fly Required Navigation Performance (RNP) approaches, a magenta 'RPA' indication time-shares with altitude in the second line of the FDB (see Fig. 3).

\section{B. Automated Terminal Proximity Alert}

$\mathrm{ATPA}^{4}$ is an advisory tool that has been added to STARS displays to help terminalarea controllers monitor separation between aircraft, improve efficiency of compression, and reduce compression errors along the final approach course. Whereas TPA operates on individual tracks manually selected by the controller, ATPA automatically identifies track pairs for qualifying aircraft. It identifies the lead and trail track, and determines the minimum required separation using each aircraft's weight class. ATPA then displays one of three types of cones originating from the trailing track: monitor (blue) cones, warning (yellow) cones, and alert (orange) cones (Fig. 4). The cones are oriented toward the lead track (i.e., they are not necessarily aligned with the trail track heading). The length of a cone corresponds to the required minimum separation for the track pair at the runway threshold.

The blue monitor cones are designed to be identical in appearance to TPA cones. ATPA

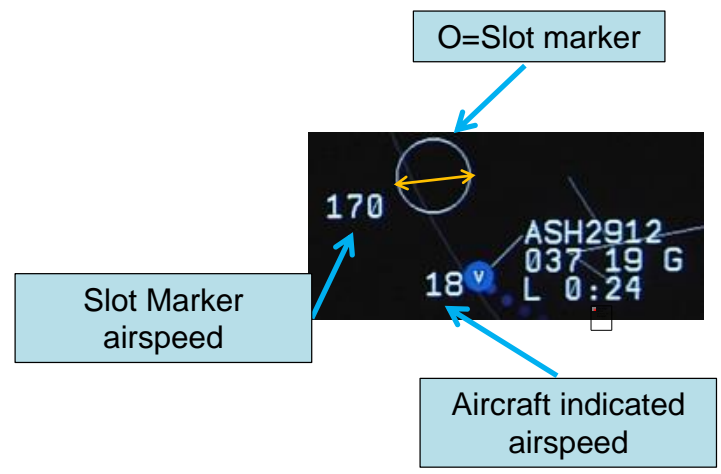

Figure 2. CMS slot marker.

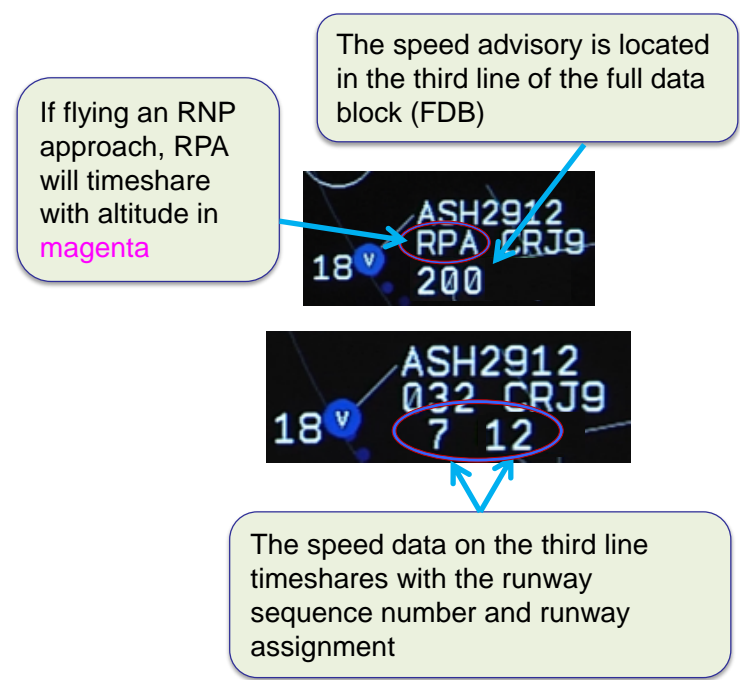

Figure 3. TSAS FDB information.

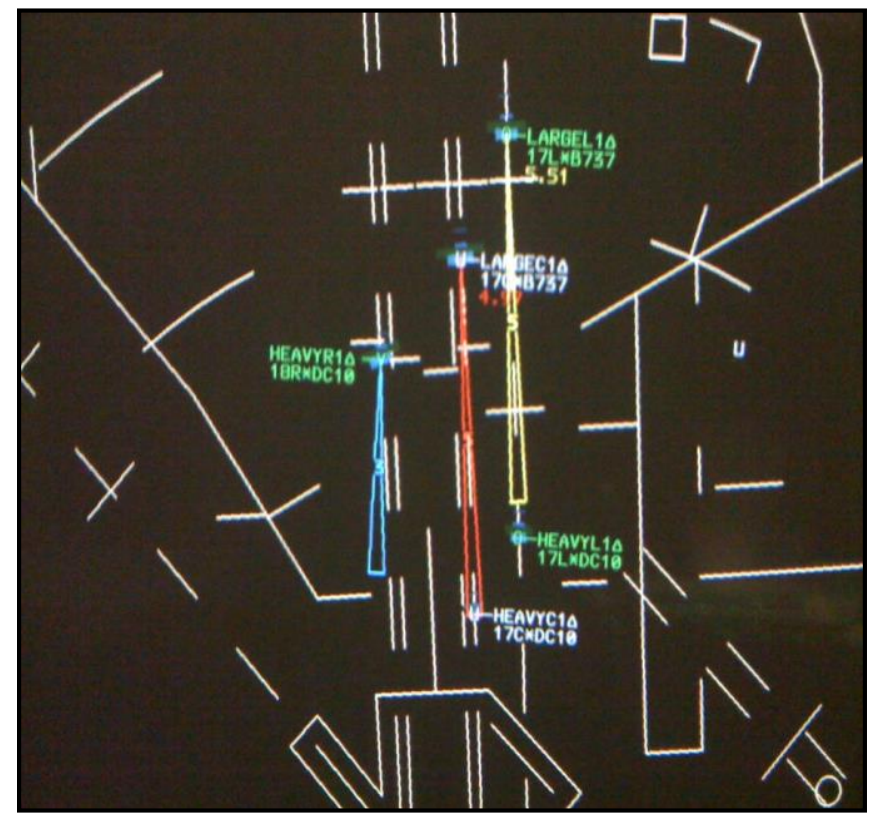

Figure 4. (From left to right) ATPA monitor, alert, and warning cones.

3

American Institute of Aeronautics and Astronautics 
displays a monitor cone on the trailing track when the distance between the the qualifying lead and trail aircraft is within $2 \mathrm{nmi}$ of the required minimum separation. If a controller has previously displayed a TPA cone of a different length, ATPA will automatically adjust the cone to the required length. ATPA displays a yellow warning cone when it predicts minimum separation will be lost in the next $45 \mathrm{~s}$ before the lead crosses the runway threshold (i.e., the previously displayed monitor cone turns yellow when ATPA predicts the loss of separation). Similarly, ATPA displays orange alert cones when it predicts minimum separation will be lost in the next $24 \mathrm{~s}$. Keyboard entries allow controllers to enable or inhibit ATPA display elements

ATPA triggers when eligible aircraft are located within a site-adapted volume (i.e., rectangle) aligned with the final approach course. Eligible aircraft must be operating under Instrument Flight Rules (IFR), with a valid track, altitude, and known weight class. A heading tolerance (e.g., 90 deg) specified for each volume determines how close the aircraft track heading must be to the final approach course for an eligible aircraft located within the volume to qualify. Arrival/departure/over-flight status and lead-track direction are also adaptable. If authorized, $2.5 \mathrm{nmi}$ reduced runway separation can be enabled for a specific volume. The distance from the threshold where the reduced separation starts is also adaptable. ATPA separation predictions use an aircraft's current ground speed when the aircraft is more than $5 \mathrm{nmi}$ from the runway threshold; within $5 \mathrm{nmi}$, ATPA computes a speed profile that reduces linearly from the current ground speed to a volume-adapted weightclass landing speed. Inside $2 \mathrm{nmi}$ to the threshold, ATPA uses the lesser of current ground speed and the weight-class landing speed. Final approach volumes may be displayed via adapted video maps.

The phase-II ATPA implementation also supports dependent-parallel runway operations, in which aircraft are additionally required to maintain $1.5 \mathrm{nmi}$ minimum diagonal distance ('stagger') from aircraft landing on the parallel runway (Fig. 5). Under staggered operations, ATPA displays both the distance to the lead track and the diagonal distance to the aircraft landing on the parallel runway in the third line of the FDB. The in-trail distance is displayed in the same color as the ATPA cone, and the stagger distance is displayed in the color of the staggered-lead aircraft's FDB.

\section{Wake-Separation Recategorization}

As new aircraft types have been introduced, the traditional wake-vortex separation matrix has become outdated, leading to excessive separation requirements between certain aircraft pairs. The former categorization of both B747 and B767 as 'heavy' jets, for example, requires a B747 to follow $4 \mathrm{nmi}$ behind a B767 when $3 \mathrm{nmi}$ is adequate to ensure safety. RECAT is the revision of required wakeseparation between certain aircraft pairs through the use of an expanded wake-separation matrix using six categories derived not solely based on aircraft weight, but also wingspan, speed, and stability characteristics. ${ }^{5}$ Fig. 6 shows

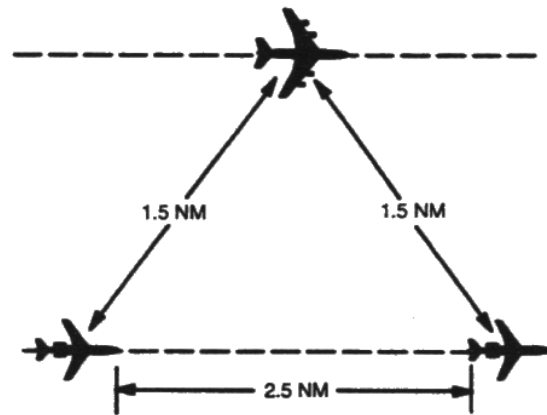

Figure 5. Diagonal separation requirements for dependent parallel operations.

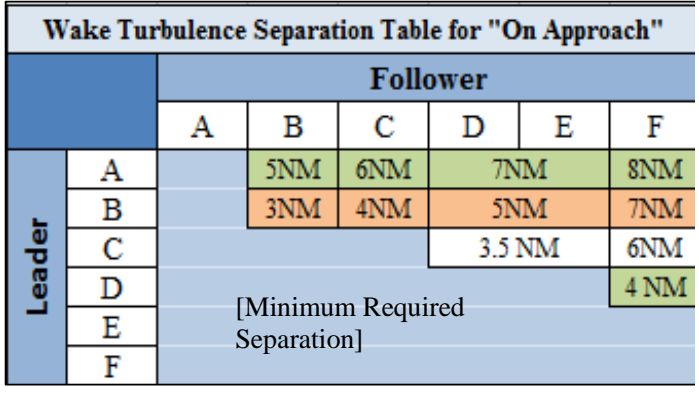

Figure 6. RECAT approach separation requirements.

the RECAT spacing for aircraft on approach. In addition to decreasing separation requirements between prevalent 'heavy' and 'large' aircraft pairs, RECAT also increases wake-separation requirements for Category ' $\mathrm{F}$ ' aircraft following aircraft in other categories. RECAT has resulted in efficiency gains in Memphis, Louisville, Cincinnati, and Atlanta, with implementation planned for more airports. ${ }^{6}$ The present work assumes that when RECAT is in use, FDBs are configured with the letter corresponding to an aircraft's RECAT category time-shared at the end of second line of the FDB.

The remainder of this paper describes the human-in-the-loop TSAS-ATPA-RECAT interoperability simulation. Section II describes the simulation method and Section III presents the results. In conclusion, the integration recommendations are summarized. 


\section{Simulation Study}

The simulation study served as a risk-mitigation measure for the FAA/NASA TSAS OIA by examining terminalarea controller-interface and interoperability issues not addressed during the OIA proper. It therefore used airspace and traffic scenarios similar to those used in the OIA and previous TSAS integration simulations. ${ }^{7}$ While the simulation scope was limited to the terminal area, center metering to the TBFM schedule as would be expected under TSAS operations was performed by confederate center controllers. This section describes the simulation.

\section{A. Airspace and Routes}

The simulation used west-flow arrivals in Phoenix TRACON (P50) airspace. The traffic transited two Feeder sectors (Apache and Quartz) and two Final sectors (Freeway and Verde), with equipped aircraft assigned to RNAV arrivals from each of four terminal-area meter-fix entry points, as shown in Fig. 7. Fig. 8 and Fig. 9 illustrate the TSAS route adaptations that provide the basis for the terminal-area scheduling and specify the nominal procedures for the slot markers and speed-advisories. Fig. 8 shows the RNAV route adaptation for arrivals to runways $25 \mathrm{~L}$ and 26 . The procedures include Required Navigation Performance (RNP) approaches for equipped aircraft. Dashed lines that appear in 'crossover' routes to the parallel arrival runway and connections to the approach indicate routes segments aircraft should fly using heading-vector instructions from controllers. Fig. 9 depicts the route adaptation

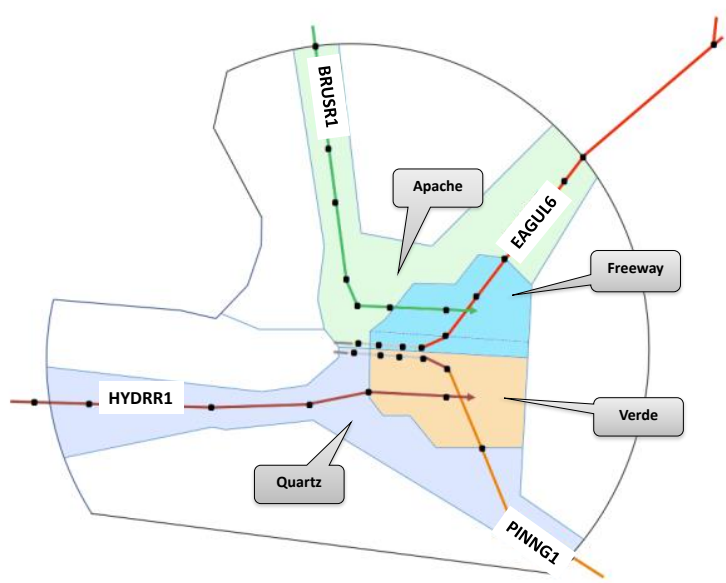

Figure 7. P50 airspace and RNAV arrivals.

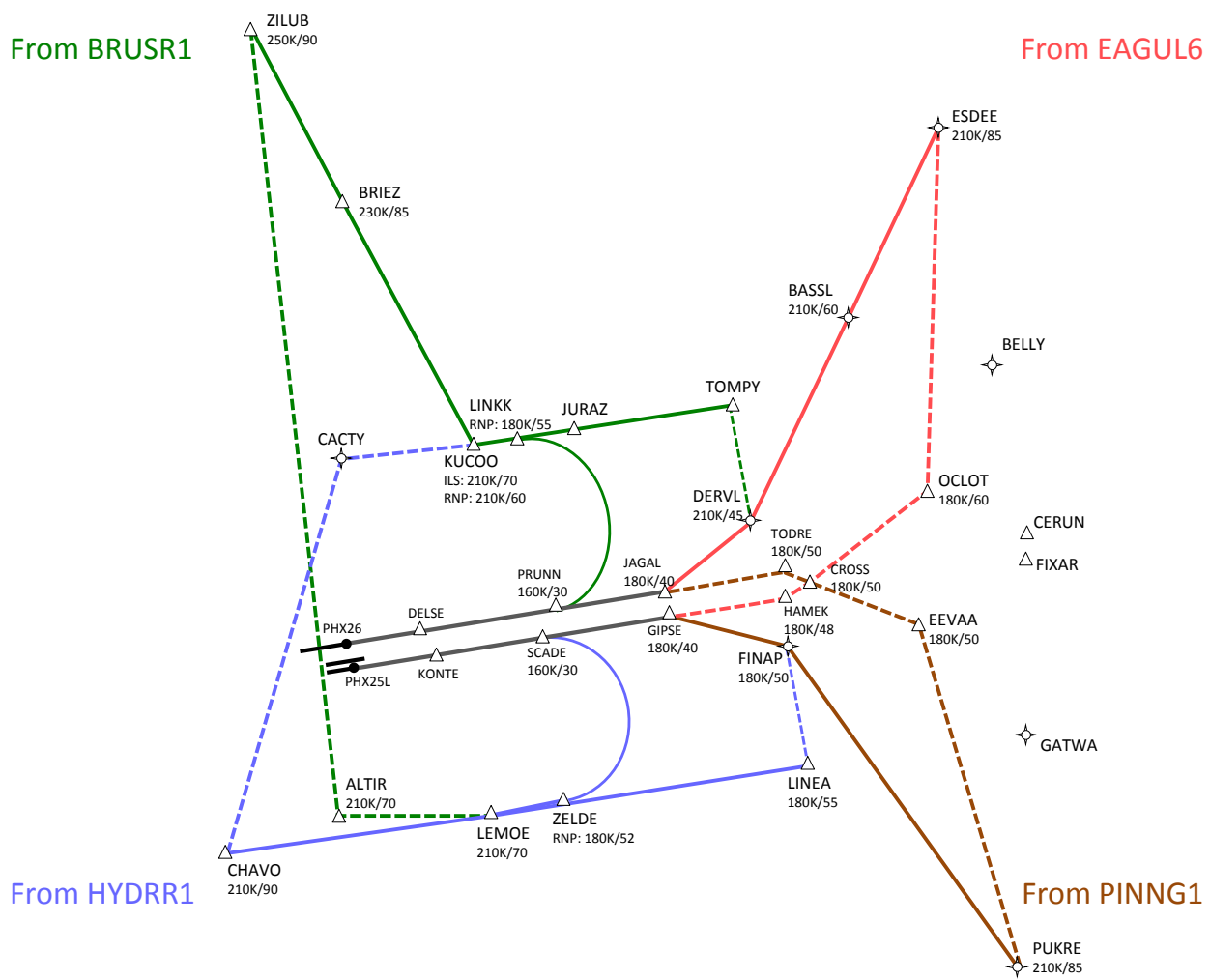

Figure 8. RNAV route adapations for arrivals to runways $25 \mathrm{~L}$ and 26. Dashed lines indicate vectoring is required. 
used for non-RNAV-equipped aircraft; controllers were also expected to vector aircraft to approximate the paths of slot markers moving along these routes.

Four different traffic scenarios were used in the simulation, two for independent-runway operations, and two for staggered dependentrunway operations. The scenarios contained between 90 and 140 aircraft and lasted approximately fifty minutes. All the scenarios included only PHX arrivals, with approximately $80 \%$ of aircraft RNAV-equipped and assigned to the RNAV arrivals. Between $32 \%$ and $43 \%$ of all arrivals were RNP equipped and assigned to the RNP approaches; controllers could cancel the RNP approaches if conditions warranted.

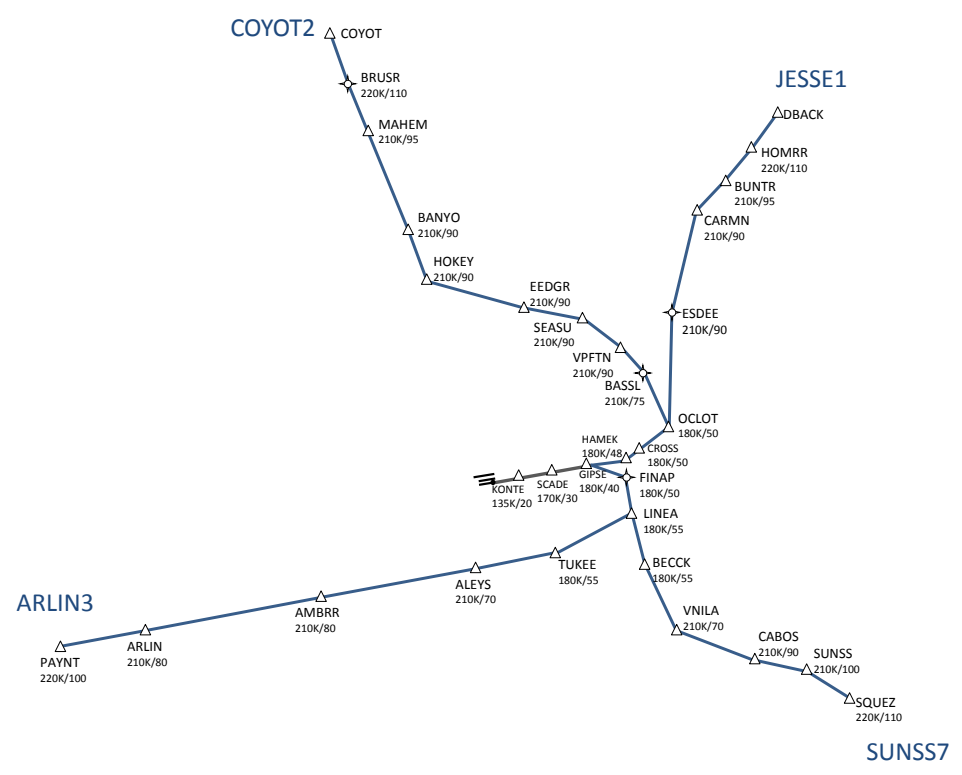

Figure 9. Adapted non-RNAV arrivals.

\section{Participants and Laboratory Environment}

To maximize the participant feedback obtainable from a one-week study, the simulation was conducted in two parallel 'worlds.' Two independent teams of TRACON controllers worked the same arrival problem in the same display condition concurrently. The 'World 1' team consisted of four local retired TRACON controllers who, as frequent subjects in the AOL, had considerable experience with the laboratory environment and the CMS tools. The 'World 2' team, on the other hand, consisted of four retired TRACON controllers from P50 who were highly experienced with the airspace but not with the tools. Both teams also included retired controller confederates who staffed Center and Tower positions. The Center confederates conditioned the arrival traffic by metering to the TSAS schedule.

The simulation platform in the AOL consisted of the Multi-Aircraft Control System (MACS) ${ }^{8}$ together with TSAS-equipped TBFM v4.2.3. ATPA was implemented within the MACS STARS emulation and configured to use RECAT wake categories; all the aircraft types in the traffic scenarios were assigned to one of the six categories. TPA was always available; controllers could use the STARS command ' $* \mathrm{P}<$ cone length $><$ aircraft $>$ ' to display a cone on any aircraft. The MACS implementation of ATPA was adapted such that track pairs were identified when both aircraft were in the adapted volume, within 90 deg of the runway heading, and the lead aircraft was assigned to the same runway. Controllers could turn TPA/ATPA monitor cones on or off at their discretion; ATPA alert and warning cones were displayed automatically and could not be switched off. RECAT was not implemented in the TBFM version used for the simulation, so that in a few cases the TSAS schedule required different spacing intervals schedule than RECAT mandates. Fig. 10 shows some photographs of the simulation components and participants.

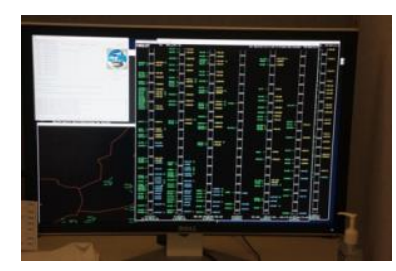

TBFM 4.2.3

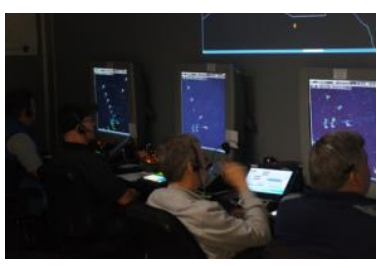

TRACON controller participants MACS-STARS

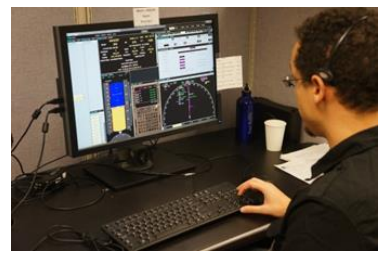

Simulation Pilot MACS-Multi Aircraft Mode

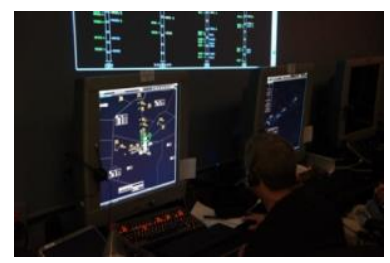

Center and Tower Confederates MACS-ERAM and MACS-STARS

Figure 10. Simulation components and participants in the Airspace Operations Laboratory. 


\section{E. Experimental Conditions}

Seven conditions were defined to capture a full range of interoperability possibilities between CMS and ATPA. In all conditions, TSAS scheduling and center metering were used, and the assigned runway, sequence number, and RECAT category were displayed in the FDB on the MACS STARS-workstation emulation. CMS tools included slot markers, speed advisories, and early/late indications, as well as schedule timelines if desired. The conditions are listed in Table 1. The first two represent the cases where only CMS or ATPA is in use, while condition 5 represents simultaneous operations. The remaining conditions represent different schemes for switching between CMS and ATPA information.

In condition 1, 'CMS only,' ATPA is turned off, and only the CMS slot markers and speed advisories or early/late indications appear. Controllers may display TPA cones at their discretion. The time-sharing scheme used in FDBs for this condition are shown in Fig. 11 for an RNP-equipped aircraft. The magenta 'RPA' and aircraft type time-share with the altitude, groundspeed, and RECAT category on the second line of the FDB, while the TSAS sequence number and runway assignement time-share on the third line with the CMS speed advisory (asynchronously with second-line information). The sequence number and runway assignment appear in yellow when the aircraft has been scheduled as a crossover to the parallel runway (see Fig. 13 and Fig. 17 below).

Condition 2, 'ATPA only,' is depicted in Fig. 12. In this condition, no slot markers, speed advisories, or early/late indications appear, but the TSAS sequence number and runway assignment remain in the third line of the FDB. In the second line, the assigned approach scratchpad information and aircraft type (not shown in Fig. 13) again time-shares with altitude, groundspeed, and RECAT category. The ATPA distance-to-lead value is appended to the third line. When
Table 1. Experimental Conditions

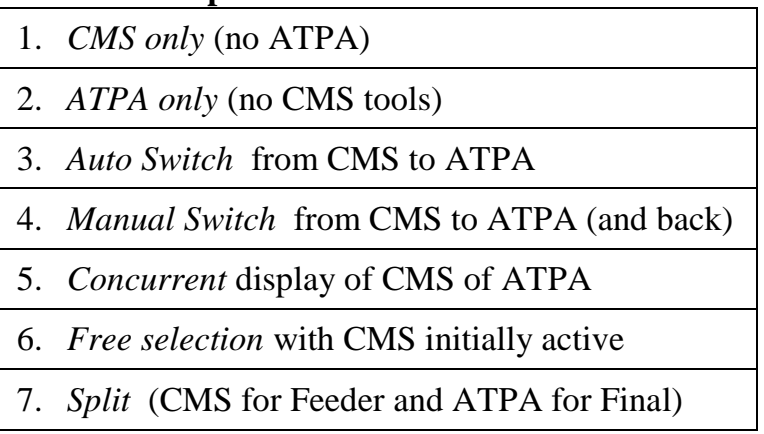

(1)

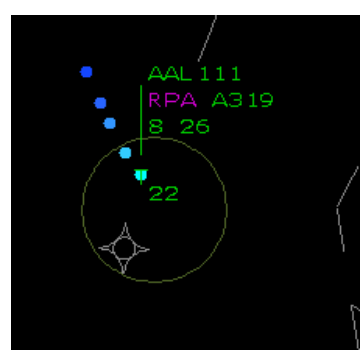

(3)

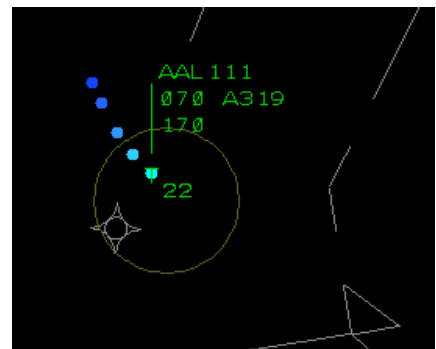

(2)

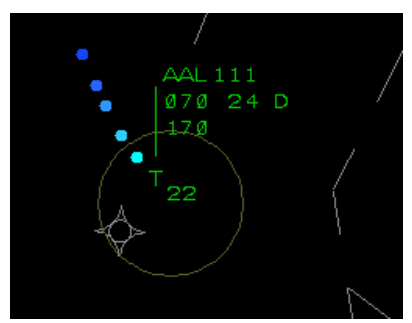

(4)

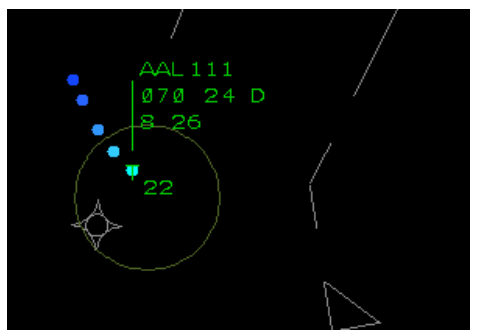

Figure 11. CMS only datablocks, with numbers indicating data block time-sharing scheme.
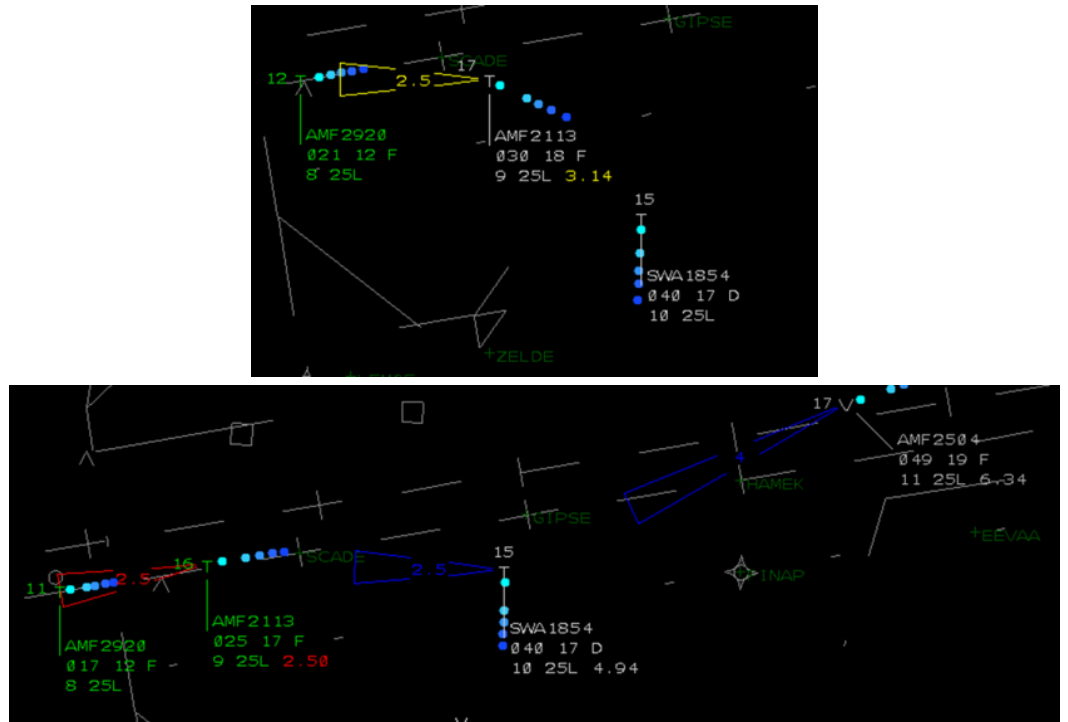

Figure 12. ATPA only, with no TSS slot markers, speed advisories, or early/ late indications. 


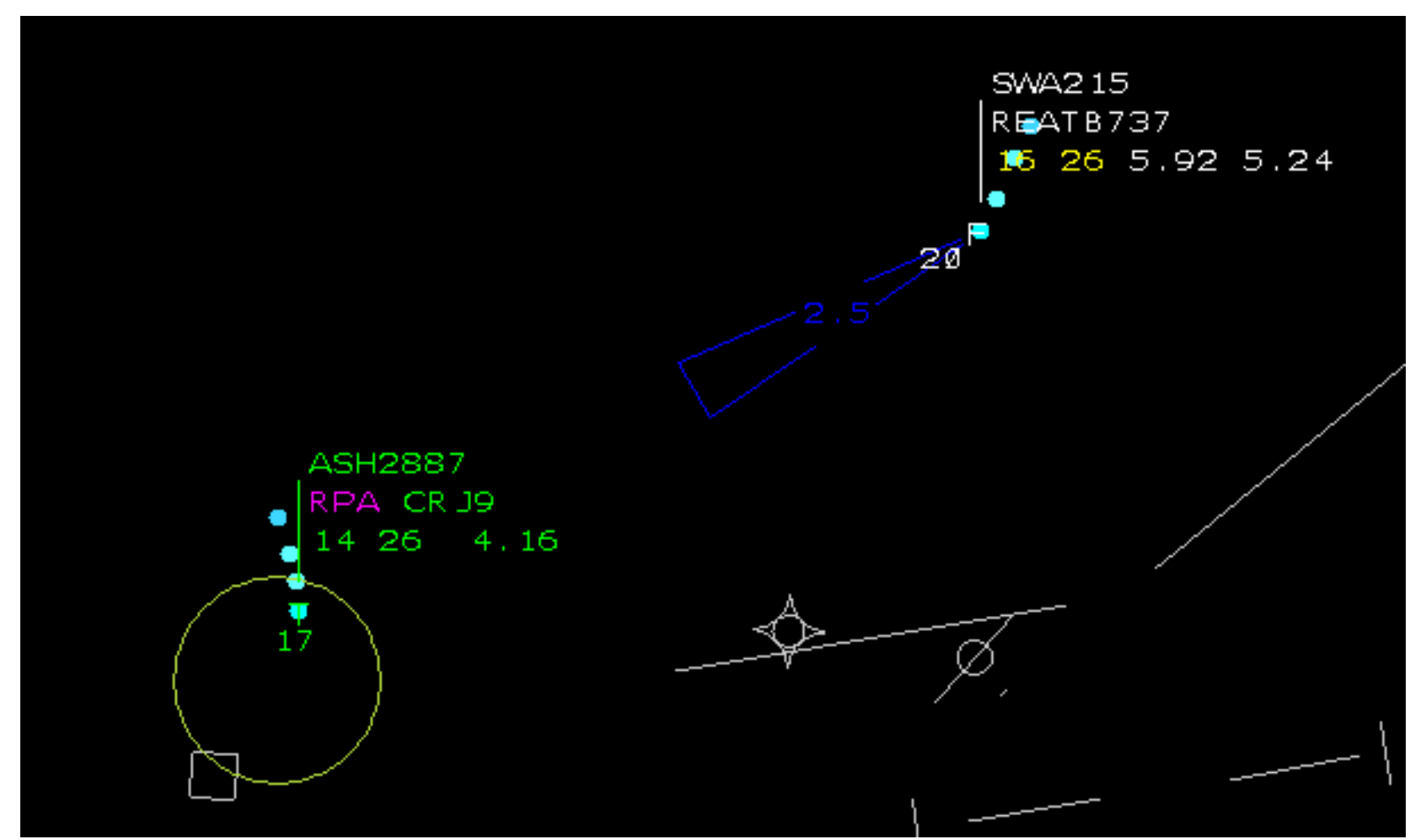

Figure 13. Auto-switch changes from CMS to ATPA when ATPA conditions are met. Yellow runway and sequence number indicates aircraft scheduled as a 'crossover.'

alert or warning ATPA cones are displayed, the distance value appears in the associated color. Controllers have the option of inhibiting the display of ATPA monitor cones.

Fig. 13 shows a display snapshot from Condition 3 ('Auto Switch') in staggered operations. In this condition the CMS tools are replaced by the ATPA information when the ATPA conditions for displaying a cone are met. Note that when ATPA identifies either the lead track, the adjacent lead on the parallel runway, or both, the corresponding distance(s) are appended to the third line of the FDB.

In Condition 4, 'Manual Switch,' (Fig. 14) FDBs initially appear with CMS information. The controller may manually change the FDB to display ATPA information, and switch a FDB back to CMS mode. When ATPA activates 'under the hood,' ATPA distance information is added to the third line of the FDB regardless of the selected mode.

The 'Concurrent' condition (Condition 5) is shown in Fig. 15. In

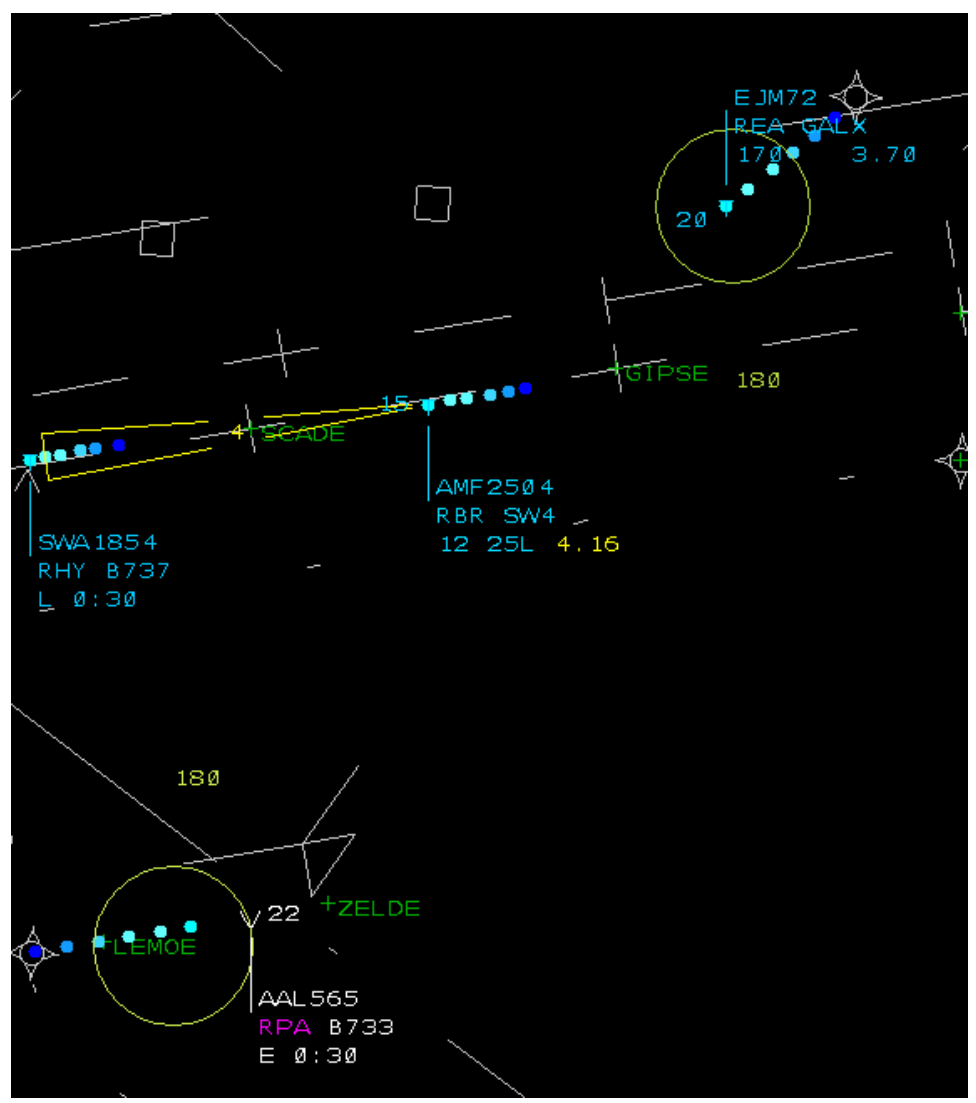

Figure 14. Display in Manual Switch condition. 
this condition, CMS information is shown for all aircraft, and as soon as ATPA activates for a particular aircraft, it is displayed with the full set of CMS and ATPA information. Controllers can choose to show ATPA, CMS tools, or nothing in Condition 6, 'Free Selection' (Fig. 16), while Condition 7 ('Split') only displays the CMS tools on the Feeder controller scopes and shows only the ATPA information on Final controller scopes (Fig. 17).

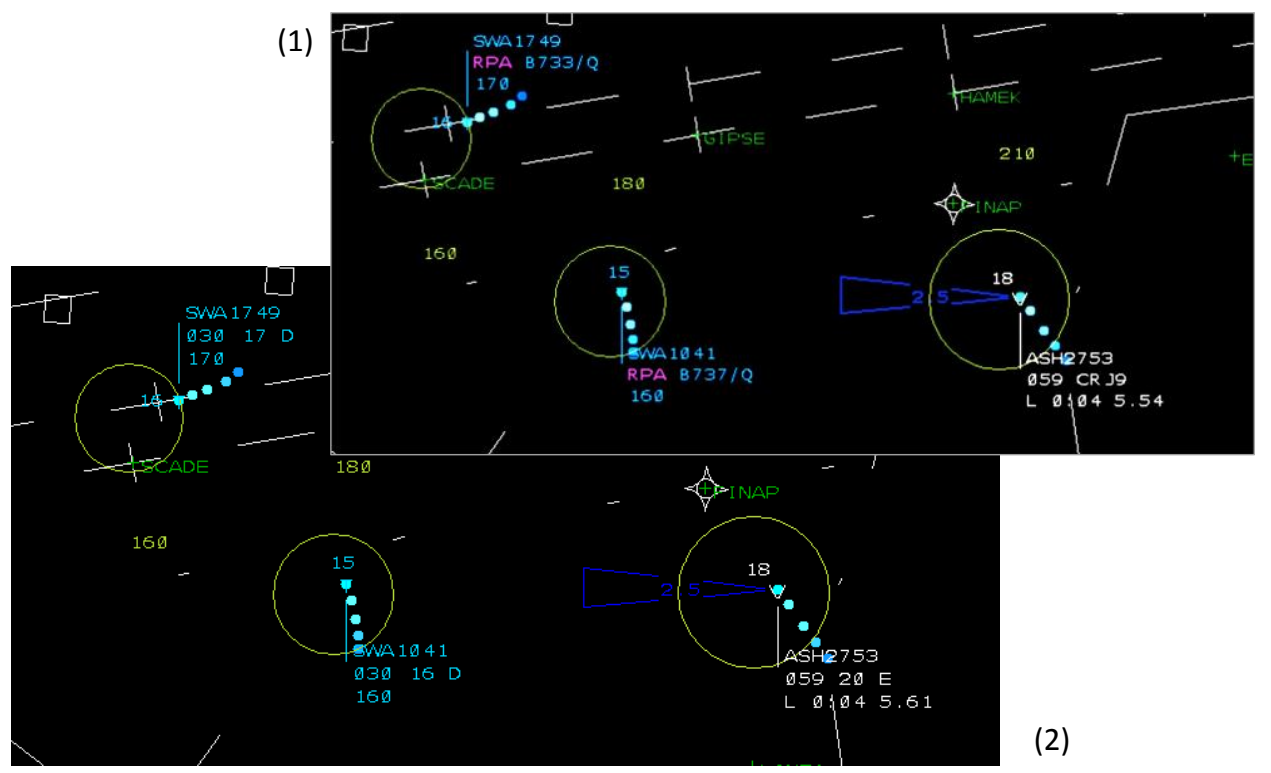

Figure 15. Concurrent display of CMS and ATPA, illustrating time-sharing scheme.

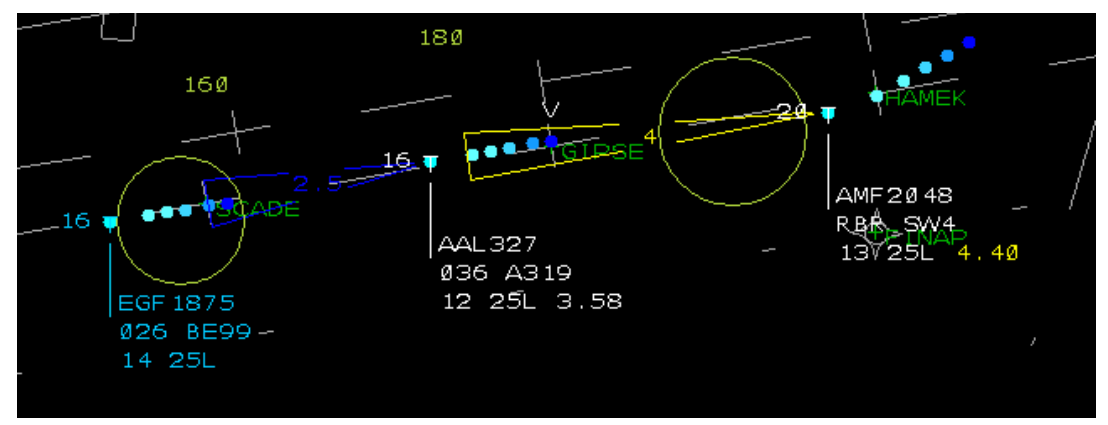

Figure 16. Free-selection condition; controllers are free to choose the displayed tools.

(1)

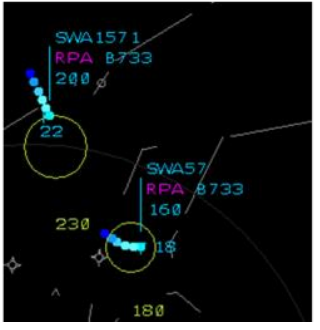

(a)

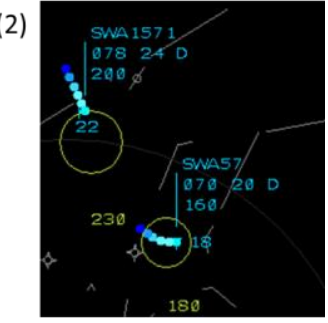

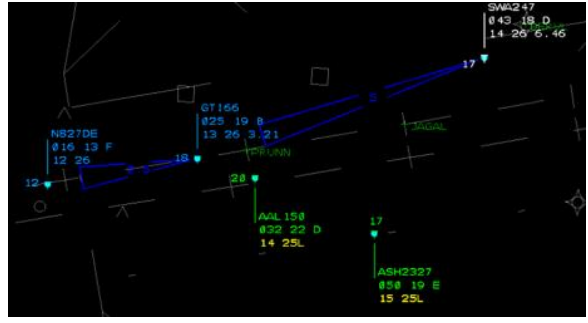

(b)

Figure 17. (a) Split condition, with only CMS tools on the Feeder display (with time sharing), and (b) only ATPA tools on the Final display. 


\section{F. Schedule and Exploratory Condition}

The study proceded according to the test schedule shown in Table 2. World 1 and World 2 followed the same schedule. To further maximize the participant feedback on each condition, the Feeder and Final controllers for each runway switched positions midway through each scenario (i.e., the north-side Feeder and north-side Final changed places, and the south-side controllers changed places.). This allowed each controller to comment on the usefulness and issues surrounding a particular display condition from the both the Feeder- and Final-controller perspectives.

Fig. 18 shows the initial rankings of the seven conditions. The rankings generally indicating a preference for more information, while keeping clutter to a minimum. In addition, usability ratings for CMS tools and ATPA indicated that both capabilities are relatively easy to learn and explore (Fig. 19). Finally, the time-sharing schemes for information in the second and third lines of the FDB were rated as 'clear' and 'fairly clear,' with little difference in mean ratings between the two simulation worlds (Fig. 20).

Following the scenarios in the initial seven conditions, all the controller participants gathered to discuss their interoperability preferences. The resulting 'exploratory' condition includes simplifications for clarity and recognizes position-specific needs (Fig. 21). First, assigned runway and sequence number are not displayed in the third line of the FDB when the ATPA separation distances appear. A standard color-coding is also applied, with in-trail separation values in blue, and diagonal separation values in green. In addition, CMS speed advisories and early/late indications do not appear for Final controllers, nor do slot markers for aircraft on the parallel runway. Speed advisories and early/late indications for Feeder controllers only appear for Feeder controllers when an aircraft's schedule error is $30 \mathrm{~s}$ or greater. Final, dwelling on any aircraft shows its slot marker, regardless of ownership. After two tests with the exploratory conditions, FDB clarity ratings increased by approximately one rating point (Fig. 22).

The following section presents the study results, including integration issues identified, subjective ratings, and RNP-approach and in-trail spacing performance.
Table 2. Test Schedule.

\begin{tabular}{|c|l|l|}
\hline Run & \multicolumn{1}{|c|}{ Scenario } & \multicolumn{1}{c|}{ Condition } \\
\hline $\mathbf{1}$ & A & CMS only/practice \\
\hline $\mathbf{2}$ & A Staggered & ATPA only/practice \\
\hline $\mathbf{3}$ & B & Concurrent/practice \\
\hline $\mathbf{4}$ & B Staggered & Auto switch \\
\hline $\mathbf{5}$ & A & Split \\
\hline $\mathbf{6}$ & A Staggered & Manual switch \\
\hline $\mathbf{7}$ & B & Free selection \\
\hline $\mathbf{8}$ & B Staggered & CMS only \\
\hline $\mathbf{9}$ & B & ATPA only \\
\hline $\mathbf{1 0}$ & A Staggered & Concurrent \\
\hline $\mathbf{1 1}$ & A & Auto switch \\
\hline $\mathbf{1 2}$ & B Staggered & Split \\
\hline $\mathbf{1 3}$ & B & Manual switch \\
\hline $\mathbf{1 4}$ & A Staggered & Free selection \\
\hline $\mathbf{1 5}$ & A & CMS only \\
\hline $\mathbf{1 6}$ & A Staggered & ATPA only \\
\hline $\mathbf{1 7}$ & B & Concurrent \\
\hline Discuss/Implement Preferred Design for Exploratory Runs \\
\hline $\mathbf{1 8}$ & A & Exploratory \\
\hline $\mathbf{1 9}$ & B Staggered & Exploratory \\
\hline & \multicolumn{2}{|l}{} \\
\hline
\end{tabular}

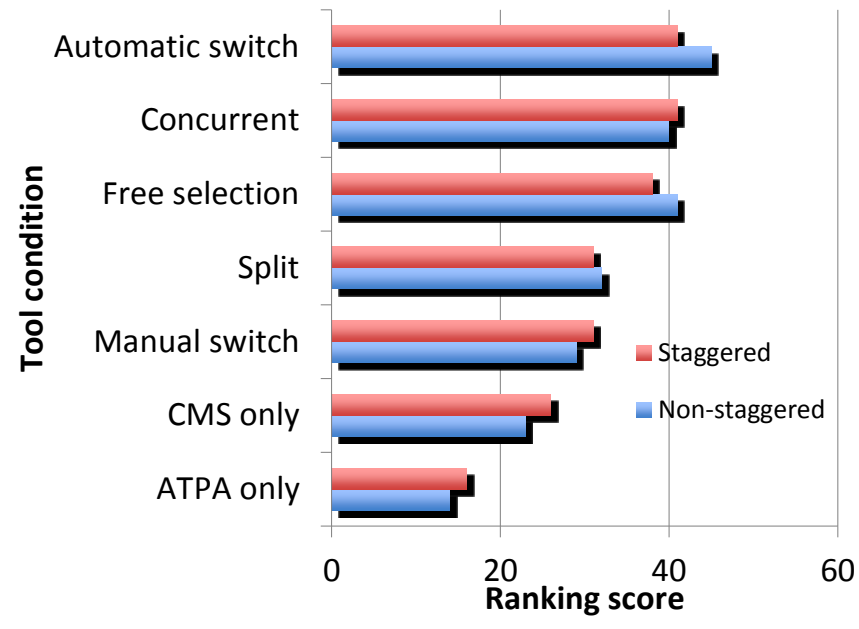

Figure 18. Initial preference rankings for the seven conditions. 


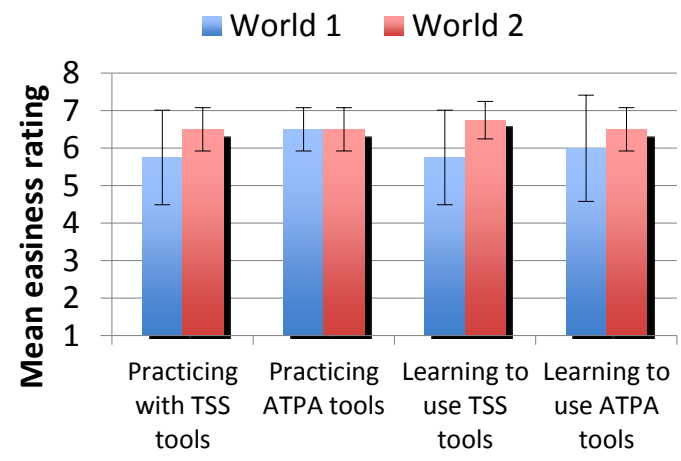

Figure 19. Ease of practicing and learning TSAS and ATPA tools.

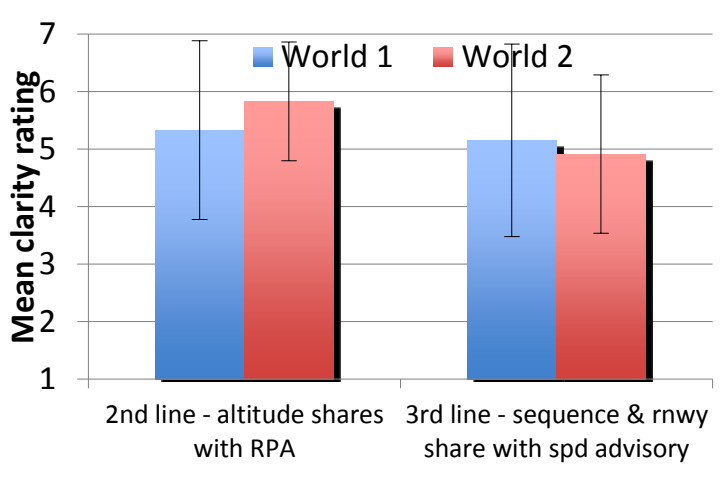

Data tag information sequence

Figure 20. Clarity of FDB time-shared information.

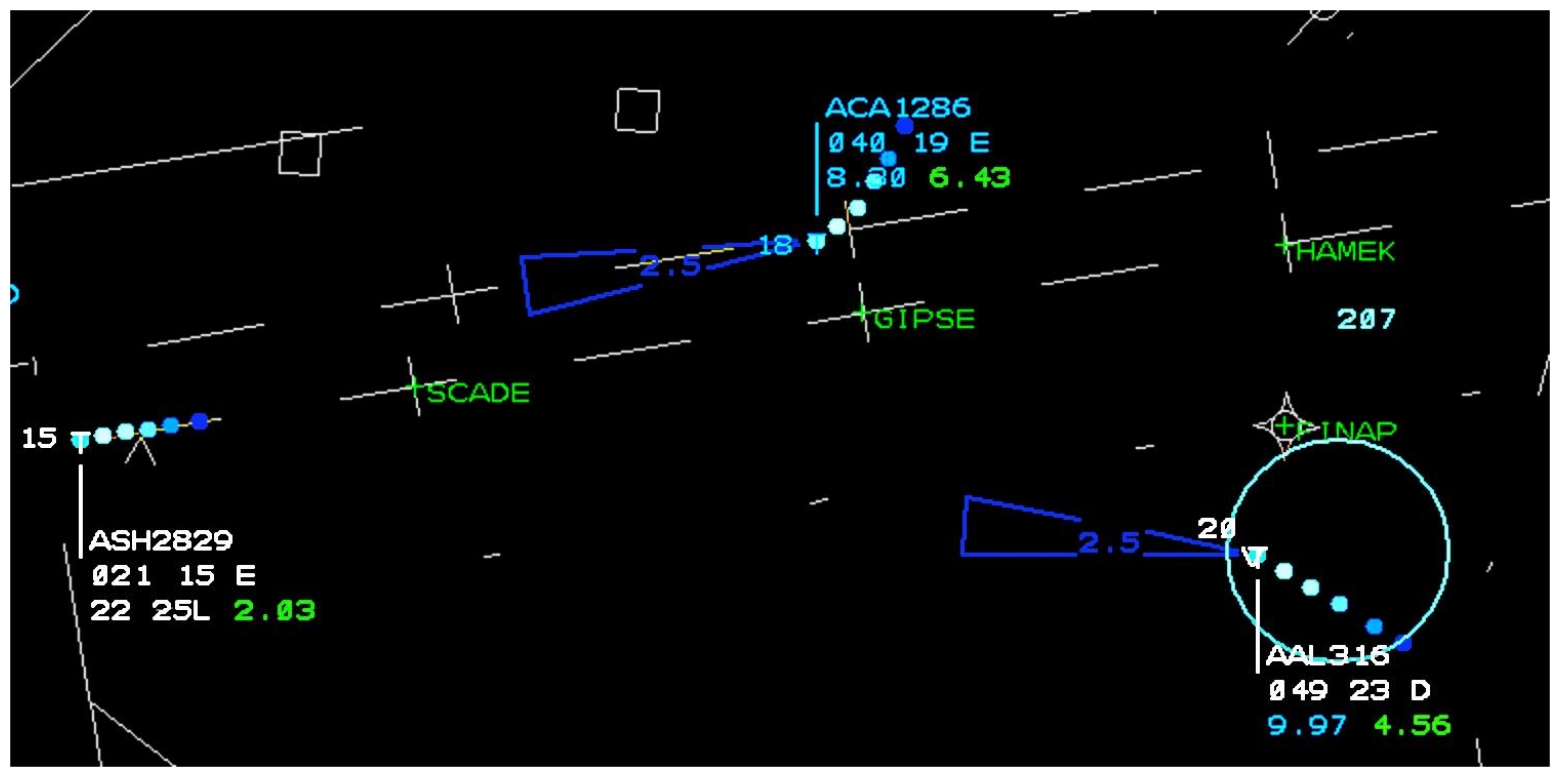

Figure 21. Exploratory condition display during staggered dependent-runway operations (with datablock dwelled to show slot marker).

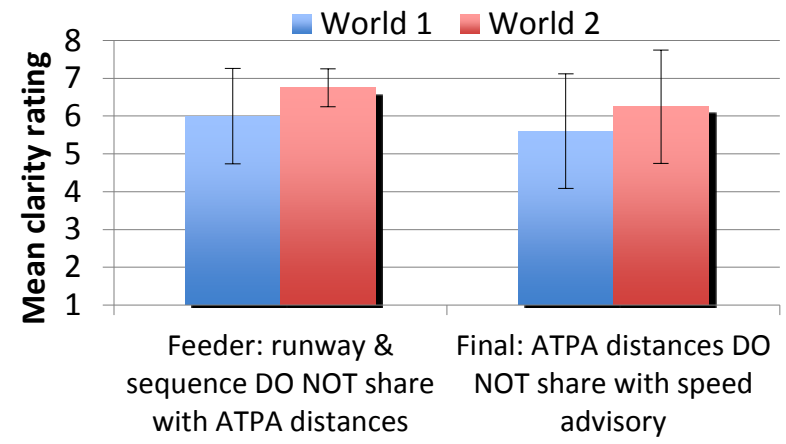

Data tag information changes

Figure 22. Improved clarity ratings for exploratory display condition. 


\section{Results}

In general the simulation showed that the new terminal-area capabilities can be effectively used together, but it also highlighted areas in which TSAS, ATPA, and RECAT integration could be strengthened. First, the MACS implementation of ATPA used the TSAS runway assignments to identify track pairs. This worked well and enabled the approach volume to be increased without increasing false alerts. However, the ATPA implementation did not use the TSAS arrival sequence; this led to cases in which ATPA initially identified the wrong track pair before switching to the correct one - typically when the actual lead aircraft was assigned to the RNP approach and entered the adapted approach volume after the non-RNP trail aircraft was already inside the volume. This situation occasionally led controllers to initially manage spacing to the wrong lead aircraft. Enabling ATPA to also use the TSAS sequence when the TSAS schedule is in use would eliminate this problem.

A second issue stems from TBFM constructing the TSAS schedule using the former wake-spacing values instead of RECAT. If the RECAT required spacing is less than that previously required, the TSAS schedule does not take advantage of reduced spacing to increase throughput; however, in the cases where RECAT requires increased separation relative to the former standards (e.g., certain cases in which the trail aircraft is Category F), the TSAS schedule does not provide adequate separation. Implementing RECAT in TSAS or at minimum, adjusting the TSAS separation matrix to ensure RECAT spacing is met, is therefore recommended.

The following subsection presents subjective results obtained from questionnaire data.

\section{G. Subjective Results}

Fig. 23 shows the average participant workload ratings for both worlds and all conditions. Mental demand was rated close to 'moderate' and time pressure was rated between 'moderate' and 'low,' while physical demand was rated 'low.' Both mean mental-demand and time-pressure ratings were highest in the ATPA-only condition and lowest in the Exploratory condition. The mean physical-demand rating was highest in the Split condition and Concurrent condition and lowest in the Exploratory condition. These differences were significant $(\mathrm{p}=0.02)$. Fig. 24 shows the average performance and effort ratings for all subjects in both worlds in all conditions. Success ratings (charted using a reversed scale) were 'high,' effort ratings were close to 'moderate,' and frustration ratings were 'low.' The average performance rating was highest for the Exploratory condition and lowest for the Split condition, the average effort rating was lowest for Manual Switch and highest for Automatic switch, and the average frustration rating was highest for ATPA Only and lowest for the Exploratory condition. These results indicate the Exploratory condition was effective for improving subjective performance while reducing workload.

The Exploratory condition also succeeded in reducing display-clutter ratings (Fig. 25), while the ratings varied between worlds for other conditions. World 1 participants rated clutter highest

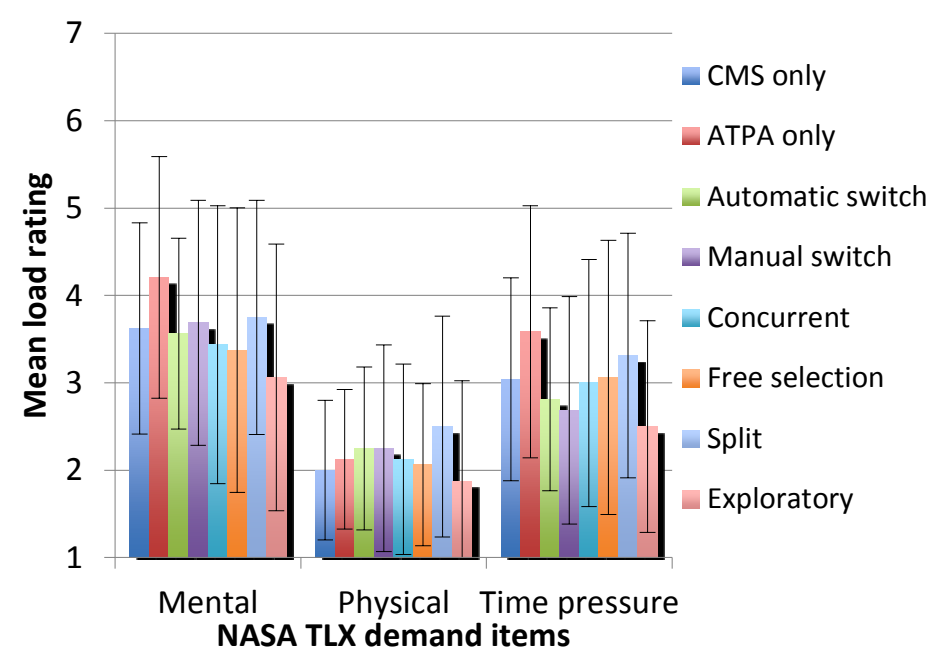

Figure 23. Task demand ratings.

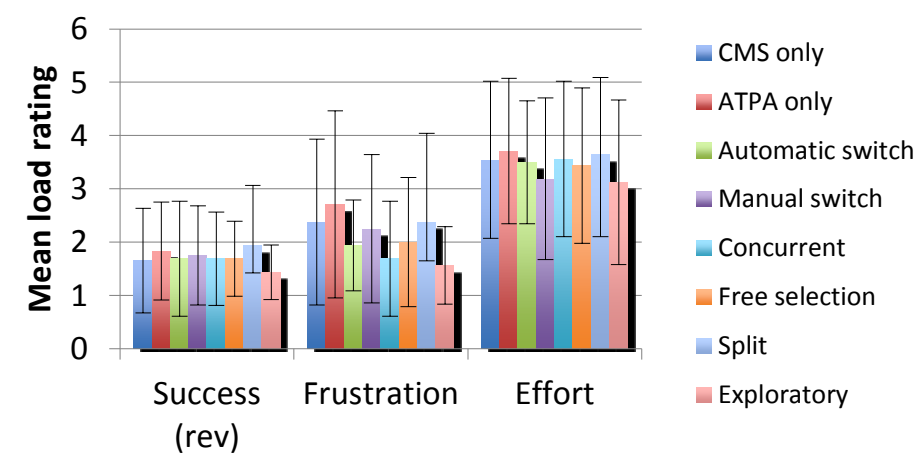

NASA TLX affect items

Figure 24. Perceived success (reversed scale), frustration, and effort ratings.

12

American Institute of Aeronautics and Astronautics 
on average in the Concurrent condition and in the Automatic switch condition, while World 2 participants rated these much lower on average, and rated both the Manual Switch and Free Selection conditions higher than the Automatic switch condition. World 1 controllers gave the Split condition their third highest clutter rating, while World 2 controllers rated it as having one of the least amounts of clutter. There was a significant difference for mean display-clutter ratings by condition $(\mathrm{p}=0.035)$. On average, participants found all conditions acceptable (Fig. 26). Considering both scales together, the Exploratory condition was the most acceptable, closely followed by Automatic Switch and Manual Switch. Differences between the mean acceptability ratings for each condition were not significant. The Exploratory condition also received the highest mean ratings on effectiveness for managing spacing (Fig. 27), while the ATPA Only condition received the lowest ratings. The differences shown in Fig. 27 are significant $(\mathrm{p}=0.001)$. Given that participant preferences were captured to greatest extent in the Exploratory condition, these results are not surprising.

After three of the simulation trials, questionnaires also sought specific feedback on the use of the CMS and ATPA tools. For the CMS tools (Fig. 28) participants reported using the CMS slot markers and FDB information for 'nearly every aircraft.' Timelines were used the least. Controllers reported consulting the speed advisories and early/late indications for 'about half the aircraft' on average. For ATPA (Fig. 29) reported using the blue monitor cone and tag information most often, for 'more than half the aircraft.' ATPA alert and warning cones were used 'for a few aircraft,' as few aircraft were predicted by ATPA to have potential separation issues during the study. Fig. 30 shows responses concerning the participants' desire for having the CMS and ATPA tools available. 57\% of the time,
Unsafe

\section{Desired Operations}

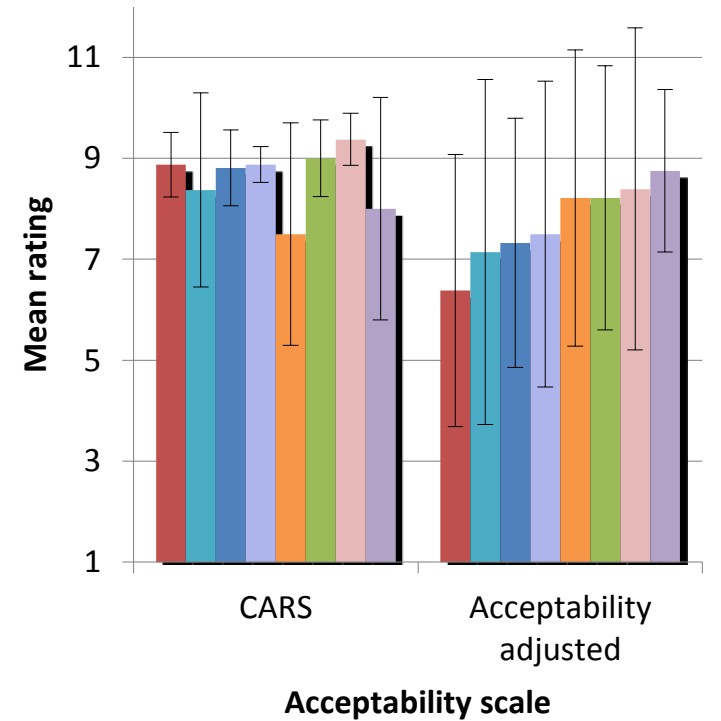

ATPA only

Concurrent

CMS only

- Split

Free selection

Automatic switch

Exploratory

Manual switch

Very acceptable

Not at all

acceptable

Figure 26. Average acceptability ratings for conditions. 


\section{7=Very Effective}

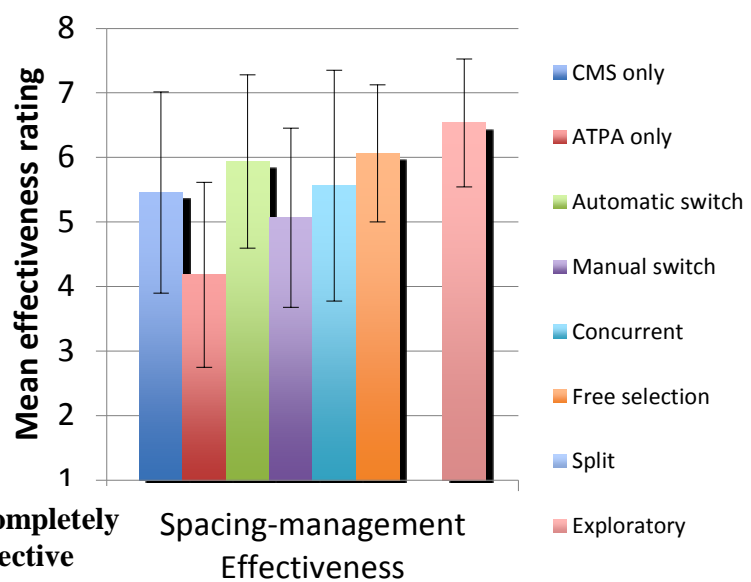

participants stated they would prefer to be able to see all the tools. Of the minority who preferred to see one toolset at a time, half expressed a desire to choose the switch point.

Tool worthiness for determining spacing and trust ratings were also collected. Fig. 31 shows that participants found having both CMS and ATPA available made determining spacing easiest, followed by CMS alone. Ratings for ATPA alone were slightly better than 'same as current day.' Average participant ratings for the tools providing safe information, confidence in the tools, tool reliability, and trust in the tools were all above 5 out of 7 , while average ratings for suspicion, wariness, and bad guidance from the tools were below 2 (Fig. 32).

Figure 27. Average effectiveness of each display condition for managing spacing.

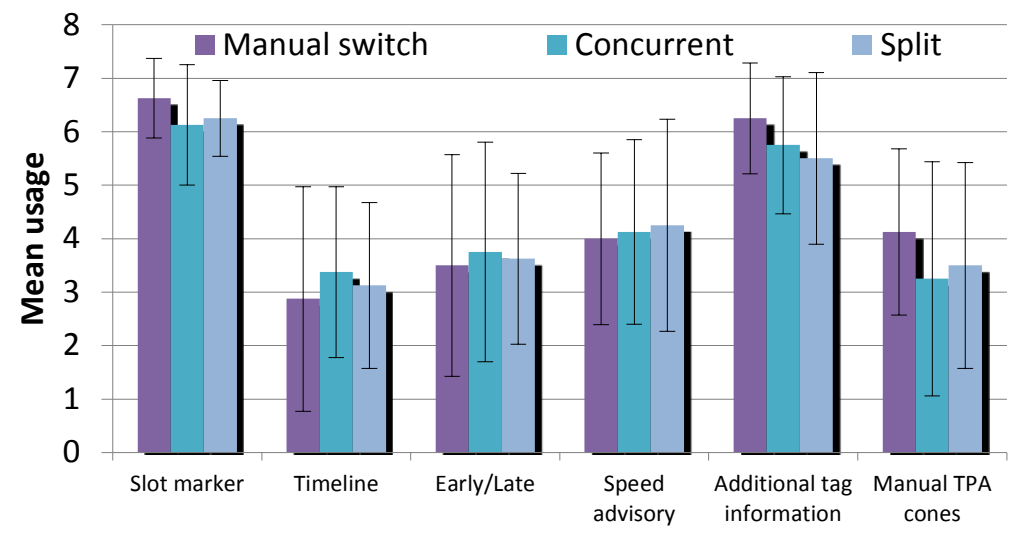

TSAS or TPA tool

Figure 28. Frequency of use of CMS tools.

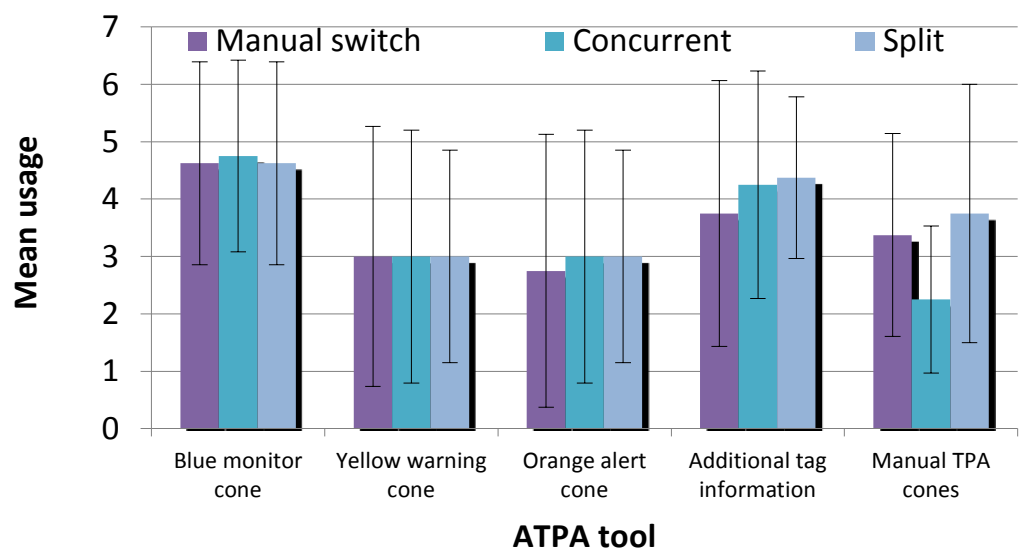

Figure 29. Frequency of use of ATPA. 


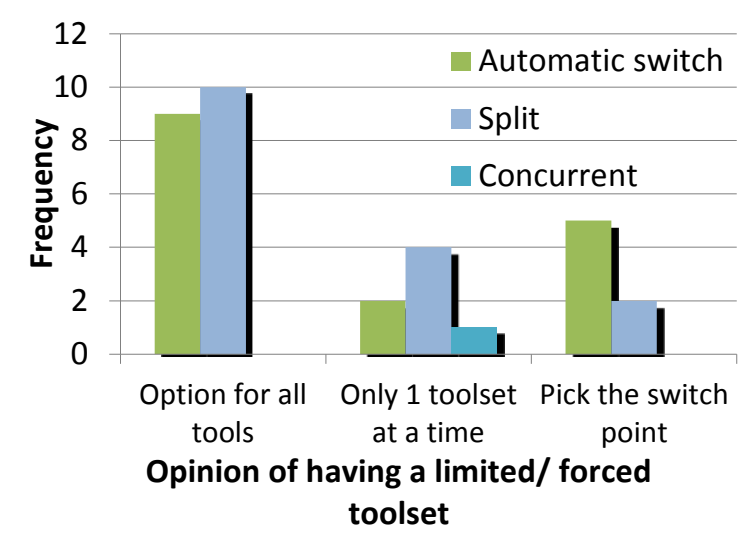

Figure 30. Preferences for all tools versus switching between CMS and ATPA.

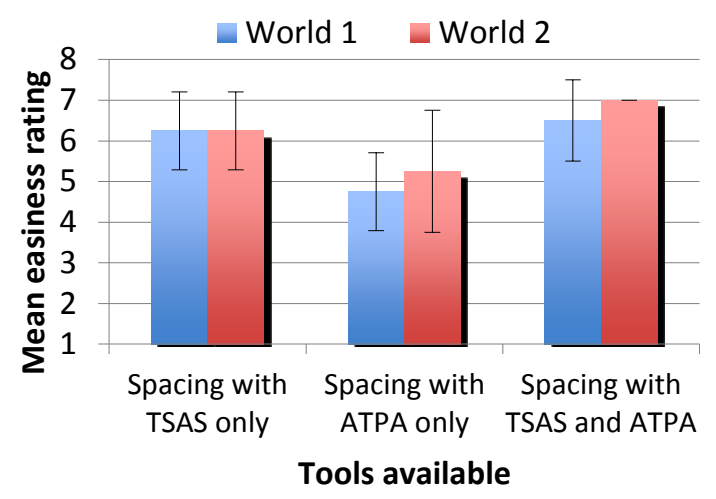

Figure 31. Ease of determining spacing using the tools (1=Much harder than current-day; 7=Much easier than current-day).

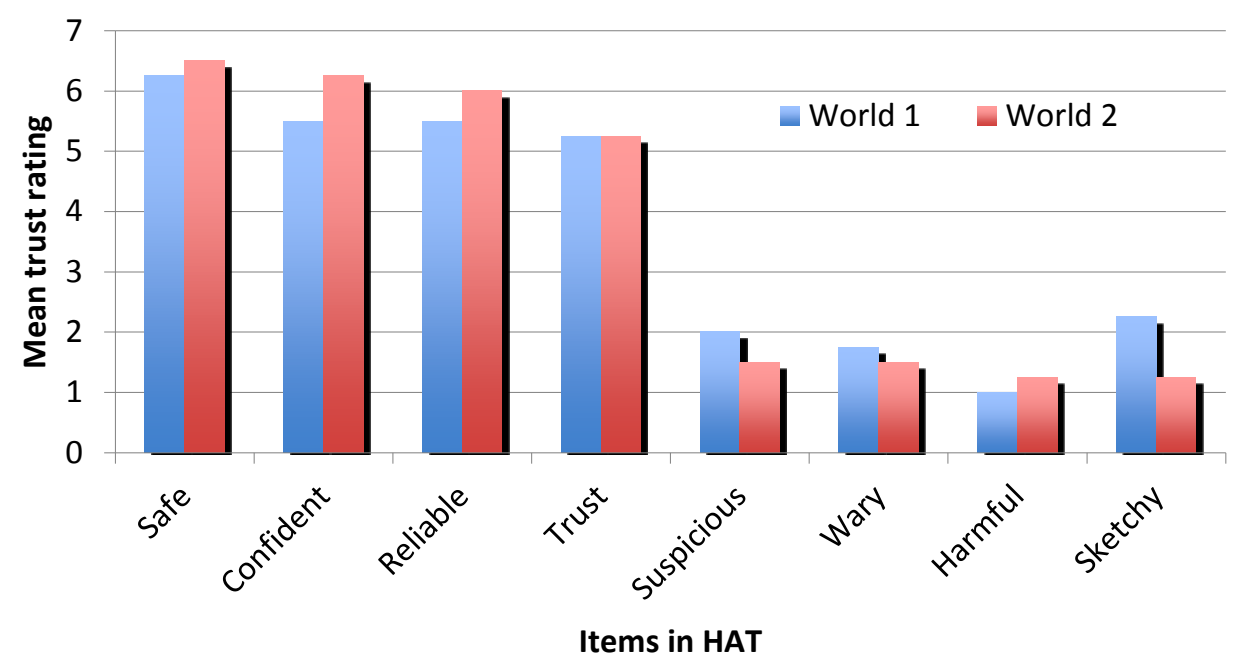

Figure 32. Average ratings for whether the tools reliably provided good information or not.

\section{H. RNP Arrivals and Final Approach Spacing}

Approximately $35 \%$ of all aircraft in the study were assigned to RNP approaches, providing an opportunity to examine the influence of tools and staggered dependent-runway operations on the RNP-approach success rate. As shown in Fig. 33, only 5\% of RNP approaches were cancelled on average, with no significant effect of independentor dependent-runway operations. The P50 TRACON controllers in World 2 were more conservative, cancelling more RNP approaches than the controllers in World 1 (Fig. 33). However, across worlds the number of successful RNP approaches was consistently high in all the study conditions (Fig. 34), indicating that with TSAS scheduling, center metering, and the runway and sequence number in the STARS datablock, high RNP-approach success rates are achievable.

Four in-trail losses-of-separation between aircraft on approach were recorded in the study, two in the Concurrent condition, one in the ATPA Only condition, and one in the Exploratory condition (Fig. 35). Three occurred in World 1 , while one occurred in World 2. None occurred during staggered operations. Close examination revealed that ATPA first identified an incorrect lead aircraft in two of the cases, while pilot and controller error contributed to the other two. 


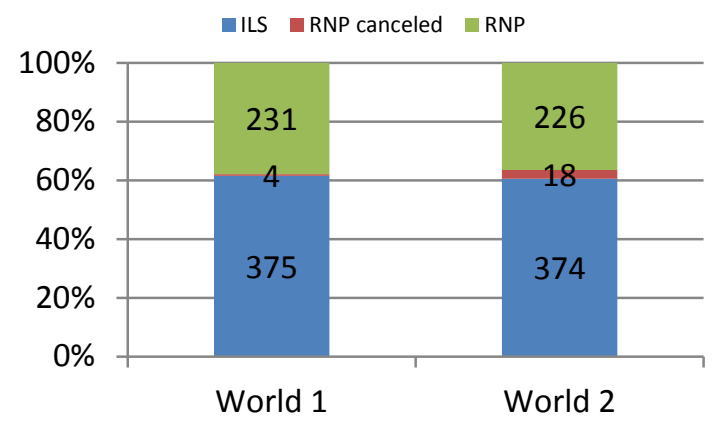

Figure 33. RNP approach cancellations by world.

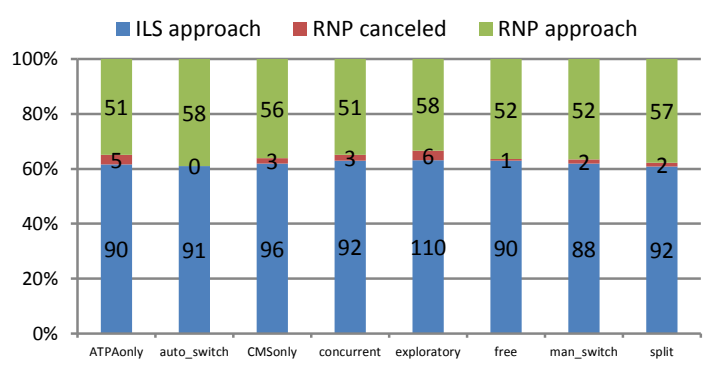

Figure 34. RNP approach cancellations by condition.

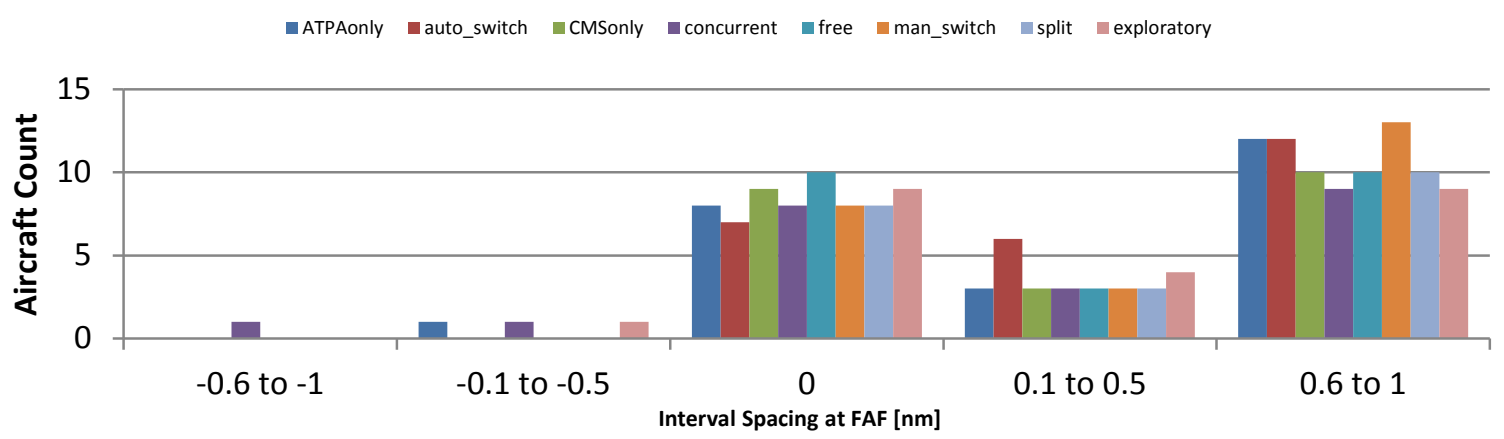

Figure 35. Histogram of in-trail spacing error at the final approach fix from -1nmi to $1 \mathrm{nmi}$ by condition.

\section{Conclusion}

The human-in-the-loop simulation tested seven potential interoperability schemes for using TSAS, ATPA, and RECAT together, as well as one developed with participant input. All the conditions were workable and acceptable. With arriving traffic conditioned according to the TSAS schedule and TSAS runway and sequence numbers available, RNP approaches were seldom cancelled, and RECAT seldom caused problems. The ATPA-only condition was least preferable. Controller participants strongly preferred having the CMS tools available on the Feeder positions, and both CMS and ATPA available on the Final positions. Controller participants liked having configuration options. The study demonstrated how display clutter can easily be reduced through a small redesign. Overall, controllers were very positive about the new integrated terminal-area capabilities.

These findings were cast as a series of specific recommendations for TSAS-ATPA-RECAT integration. The recommendations are:

- Use TSAS scheduling for center metering and TSAS runway and sequence numbers in the TRACON to organize complex TRACON traffic flows (e.g., flows that include RNP arrivals).

- Make CMS slot markers available on both Feeders and Final controller positions. Speed advisories and early/late indications should be optional for Final controllers.

- Reduce clutter by making certain information available upon dwell only (e.g., slot markers for arrivals to a parallel runway, speed advisories).

- $\quad$ ATPA is useful on Final positions; CMS-ATPA switch-point should be adaptable/configurable.

- Revisit alert times for ATPA; in some situations, more lead time may be needed.

- If controllers are working toward the TSAS schedule, consider also using TSAS runway and sequencenumber information to filter ATPA track pairs.

- Implement RECAT in TSAS; if RECAT is not fully implemented, ensure TSAS spacing is equal to or greater than RECAT spacing. 


\section{Acknowledgments}

This work was supported by the NASA ATD-1 activity, John Robinson, Chief Engineer. Thanks to all the FAA personnel who attended the study and provided feedback on the recommendations.

\section{References}

${ }^{1}$ Prevot, T., Baxley, B., Callantine, T., Johnson, W., Quon, L., Robinson, J., and Swenson, H., "NASA's ATM Technology Demonstration-1: Transitioning Fuel Efficient, High Throughput Arrival Operations from Simulation to Reality," Proceedings of the International Conference on Human-Computer Interaction in Aerospace (HCI-Aero 2012), Brussels, September 2012.

${ }^{2}$ Robinson III, J. E., Thipphavong, J., and Johnson, W. C., "Enabling Performance-Based Navigation Arrivals: Development and Simulation Testing of the Terminal Sequencing and Spacing System," Proceedings of the 11th FAA/Europe ATM R\&D Seminar (ATM2015), Lisbon, June 2015.

${ }^{3}$ Prevot, T., "How to Compute a Slot Marker-Calculation of Controller-Managed Spacing Tools for Efficient Descents with Precision Scheduling," Proceedings of the 31st Digital Avionics Systems Conference, Williamsburg, VA, October 2012.

${ }^{4}$ Functional Description Narrative N32422, “Automated Terminal Proximity Alert (ATPA)-Final Approach Course,” ATO05CARTS-1055, US Department of Transportation/Federal Aviation Administration, Washington, DC.

${ }^{5}$ Flight Standards Service, Safety Alert for Operators 12007, US Department of Transportation/Federal Aviation Administration, Washington, DC, 22 October 2013.

${ }^{6}$ Federal Aviation Administration, "Fact Sheet-Wake RECAT," 21 April, $2015 . \quad$ URL: https://www.faa.gov/fact_sheets/news_story.cfm?newsid=18676 [cited: 27 April 2016].

${ }^{7}$ Callantine, T., Kupfer, M., Martin, L., Mercer, J., and Prevot, T., "System-Level Performance Evaluation of ATD-1 GroundBased Technologies," Proceedings of the 14th AIAA Aviation Technology, Integration, and Operations Conference, Atlanta, 1620 June 2014.

${ }^{8}$ Prevot, T., Smith, N., Palmer, E., Callantine, T., Lee, P., Mercer, J., Martin, L., Brasil C., and Cabrall, C., “An Overview of Current Capabilities and Research Activities in the Airspace Operations Laboratory at NASA Ames Research Center," Proceedings of the 14th AIAA Aviation Technology, Integration, and Operations Conference, Atlanta, 16-20 June 2014. 